Revista de Matemática: Teoría y Aplicaciones 3(1): 45-60 (1996)

\title{
PRODUCTOS DE KRONECKER
}

\author{
Jorge Poltronieri VArgas ${ }^{1}$
}

\begin{abstract}
Resumen
En el estudio de las formas cuadráticas hemos obtenido fórmulas para el cálculo de covarianzas. En este trabajo realizamos un estudio sistemático de los productos que llamamos de Kronecker y que serán de gran ayuda para estos cálculos. Se introducen el producto de Kronecker asimétrico y antisimétrico.
\end{abstract}

\begin{abstract}
We obtain the formulae of covariances between random, introducting the Kronecker's products: asymmetrical and antisymmetrical.
\end{abstract}

\section{Estudio de los productos de Kronecker}

Nuestro interés es el de definir diferentes productos de Kronecker, que serán de gran utilidad para el cálculo de covarianzas.

Definición 1 Sean $A_{n \times m}$ y $B_{p \times q}$ matrices, se define por el producto simétrico de Kronecker la matriz denotada $A \otimes B$ de tamaño $n p \times m q$ tal que la entrada ijkl (entrada $k l$ del bloque $i j)$ es $(A \otimes B)_{i j k l}=a_{i j} b_{k l}$, donde $1 \leq i \leq n, 1 \leq j \leq m, 1 \leq k \leq p, 1 \leq l \leq q$. donde

En general se puede definir $A \otimes B$ de otra manera, $\left(A_{n \times m} \otimes B_{p \times q}\right)_{\alpha, \beta=p(i-1)+k, q(j-1)+l}$,

$$
\left(A_{n \times m} \otimes B_{p \times q}\right)_{\substack{\alpha, \beta=p(i-1)+k \\ 1 \leq i \leq n \\ n p \times m q}} \underset{\substack{q(j-1)+l \\ 1 \leq k \leq m \\ 1 \leq l \leq q}}{1 \leq l \leq q}
$$

Definición 2 Sean $A_{n \times m}$ y $B_{p \times q}$ matrices, se define por el producto asimétrico de Kronecker la matriz denotada $A \dot{\otimes} B$ de tamaño $n p \times m q$ tal que la entrada ijkl (entrada $k l$ del bloque ij) es $(A \dot{\otimes} B)_{i j k l}=a_{i l} b_{k j}=(A \otimes B)_{i l k j}$, es decir que en la entrada ijkl de $A \dot{\otimes} B$ se encuentra la entrada ilkj de $A \otimes B$, donde $1 \leq i \leq n, 1 \leq j \leq q, 1 \leq k \leq p$, $1 \leq l \leq m$.

\footnotetext{
${ }^{1}$ Escuela de Matemática, Universidad de Costa Rica, 2060 San José, Costa Rica
} 
Similarmente se puede definir

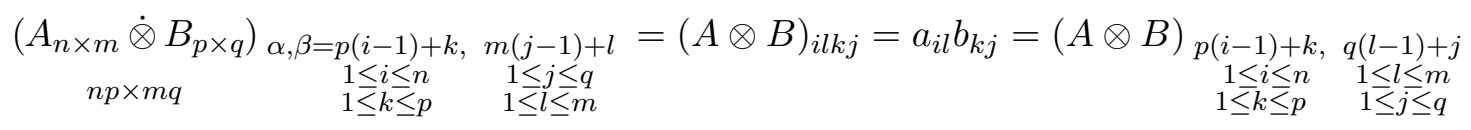

Definición 3 Sean $A_{n \times m}$ y $B_{p \times q}$ matrices, se define por el producto anti-simétrico de Kronecker la matriz denotada $A \ddot{\otimes} B$ de tamaño $n m \times p q$ tal que la entrada ijkl (entrada $k l$ del bloque $i j)$ es $(A \ddot{\otimes} B)_{i j k l}=a_{i k} b_{j l}=(A \otimes B)_{i k j l}$, es decir que en la entrada ijkl de $A \ddot{\otimes} B$ se encuentra la entrada ikjl de $A \otimes B$, donde $1 \leq i \leq n, 1 \leq j \leq p, 1 \leq k \leq m$, $1 \leq l \leq q$.

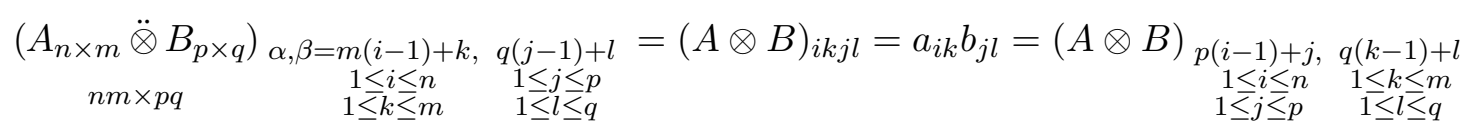

Algunas propiedades de estas matrices son

- $(A \otimes B)^{\prime}=A^{\prime} \otimes B^{\prime}$

- $(A \dot{\otimes} B)^{\prime}=B^{\prime} \dot{\otimes} A^{\prime}$

- $(A \ddot{\otimes} B)^{\prime}=B \ddot{\otimes} A$.

Sabemos que $X=\left(X_{1}, \ldots, X_{p}\right)$, entonces se define $[X]=\left[\begin{array}{c}X_{1} \\ \vdots \\ X_{p}\end{array}\right]_{n p \times 1} \quad$ o sea que la matriz $X$ se transforma en un vector colocando todos los vectores $X_{i}$ en columna. Así tenemos

- $[B X C]=C^{\prime} \otimes B[X]$.

- $[A][B]^{\prime}=A^{\prime} \ddot{\otimes} B^{\prime}$.

- $\operatorname{tr}\left(B X^{\prime} C X D\right)=\left[X^{\prime}\right]^{\prime} B^{\prime} D^{\prime} \otimes C[X]=\left[X^{\prime}\right]^{\prime} D B \otimes C^{\prime}[X]$

- $[B C]=I \otimes B[C]=C^{\prime} \otimes I[B]=C^{\prime} \otimes B[I]$

- $\left[B^{\prime}\right]^{\prime}(I \otimes C)[D]=1 \ddot{\otimes} B I \otimes C D \ddot{\otimes} 1=1 \ddot{\otimes} B D^{\prime} C^{\prime} \ddot{\otimes} 1=\operatorname{tr}(B C D) \ddot{\otimes} 1=\operatorname{tr}(B C D)$

- $\left[x y^{\prime}\right]=y \otimes x, \quad z y^{\prime}=y^{\prime} \otimes z=z \otimes y^{\prime}$

- $x y^{\prime}=x \otimes y^{\prime}=x \ddot{\otimes} y^{\prime}=y^{\prime} \otimes x=x^{\prime} \ddot{\otimes} y$

- $y q^{\prime} \otimes x z^{\prime}=y x^{\prime} \ddot{\otimes} q z^{\prime}=y z^{\prime} \dot{\otimes} x q^{\prime}$

- $x y^{\prime} \ddot{\otimes} z q^{\prime}=x z^{\prime} \otimes y q^{\prime}=\left[y x^{\prime}\right]\left[q z^{\prime}\right]^{\prime}$.

- $x \otimes y z^{\prime}=x 1^{\prime} \otimes y z^{\prime}=x y^{\prime} \ddot{\otimes} 1 z^{\prime}=x y^{\prime} \ddot{\otimes} z^{\prime}$ 
Otras propiedades interesantes son las siguientes.

- Si $A$ es $m \times m$ con valores propios $\left\{a_{1}, \ldots, a_{m}\right\}$ y vectores propios $\left\{u_{1}, \ldots, u_{m}\right\}$ y $B$ es $n \times n$ con valores propios $\left\{b_{1}, \ldots, b_{n}\right\}$ y vectores propios $\left\{v_{1}, \ldots, v_{n}\right\}$, entonces $u_{i} \otimes v_{j}$ es un vector propio de $A \otimes B$ de valor propio $a_{i} b_{j}$.

- Sean $\left\{u_{i} / i=1, \ldots, n\right\},\left\{v_{i} / i=1, \ldots, m\right\},\left\{f_{i} / i=1, \ldots, p\right\},\left\{s_{i} / i=1, \ldots, q\right\}$ bases canónicas de los espacios $\mathbb{R}^{n}, \mathbb{R}^{m}, \mathbb{R}^{p}, \mathbb{R}^{q}$ respectivamente. Sea $H_{i j}=u_{i} t_{j}^{\prime}=u_{i} \otimes t_{j}^{\prime}$ (respectivamente $J_{k l}=v_{k} s_{l}^{\prime}, R_{k j}=v_{k} t_{j}^{\prime}, L_{i l}=u_{i} s_{l}^{\prime}$ ), la matriz $n \times p$ es tal que la entrada $i j$ vale 1 y las demás $0, i=1, \ldots, n, j=1, \ldots, p$. El conjunto $\left\{H_{i j} / i=\right.$ $1, \ldots, n ; j=1, \ldots, p\}$ es la base canónica de $L\left(\mathbb{R}^{n}, \mathbb{R}^{p}\right)$.

Sea $K=\sum_{i=1}^{n} \sum_{j=1}^{p} H_{i j} \otimes H_{i j}^{\prime}$, matriz $n p \times n p$ que denotamos $K_{n p}$. La matriz $K^{\prime}$ aunque tiene la misma dimensión que $K$, es preferible denotarla $K_{p n}^{\prime}$ para tener mayor claridad en las aplicaciones.

Observación Podemos definir a partir de la matriz $M_{n p \times m q}$, las matrices $\dot{M}_{n p \times m q} \mathrm{y}$ $\ddot{M}_{n m \times p q}$ de la siguiente manera

$$
\dot{M}_{i j k l}=M_{i l k j}, \quad \ddot{M}_{i j k l}=M_{i k j l} .
$$

Se puede probar que si $M=A \otimes B$, entonces $\dot{M}=A \dot{\otimes} B$ y $\ddot{M}=A \ddot{\otimes} B$.

Observemos que si $M=\left(\begin{array}{ccc}M^{11} & \cdots & M^{1 m} \\ \vdots & \ddots & \vdots \\ M^{n 1} & \cdots & M^{n m}\end{array}\right), M_{i j k l}=M_{k l}^{i j}$ y además $M K=M \sum_{s t} J^{s t} \otimes$ $J^{s t \prime}$, i.e.

$$
\begin{aligned}
(M K)_{\alpha \beta} & =\sum_{\gamma s t} M_{\alpha \gamma}\left(J^{s t} \otimes J^{t s}\right)_{\gamma \beta} \\
& =\sum_{j l s t} M_{k l}^{i j}\left(J^{s t}\right)_{j u}\left(J^{t s}\right)_{l v} \\
& =\sum_{j l s t} M_{k l}^{i j} \delta_{s j} \delta_{t u} \delta_{t l} \delta_{s v} \\
& =\sum_{l s t} M_{k l}^{i s} \delta_{t u} \delta_{t l} \delta_{s v} \\
& =\sum_{s t} M_{k t}^{i s} \delta_{t u} \delta_{s v}=M_{k u}^{i v}=M_{i v k u} \\
& =\dot{M}_{i u k v}=\dot{M}_{\alpha \beta},
\end{aligned}
$$

o sea $M K=\dot{M}$. 


$$
\begin{aligned}
& K_{n p}=K_{p n}^{\prime}=\sum_{j=1}^{p} \sum_{i=1}^{n} H_{i j} \otimes H_{i j}^{\prime}=\sum_{j=1}^{p} \sum_{i=1}^{n} H_{i j}^{\prime} \otimes H_{i j} \quad K_{m q}=K_{q m}^{\prime}=\sum_{l=1}^{q} \sum_{k=1}^{m} J_{k l} \otimes J_{k l}^{\prime} \\
& K=K^{\prime}=K^{-1} \\
& K_{n p} K_{p n}^{\prime}=I_{n p}=I_{n} \otimes I_{p}=I_{p} \otimes I_{n} \\
& K_{m q} K_{q m}^{\prime}=I_{m q}=I_{m} \otimes I_{q}=I_{q} \otimes I_{m} \\
& K_{n q}=\sum_{s=1}^{n} \sum_{t=1}^{q} L_{s t} \otimes L_{s t}^{\prime} \\
& K_{n p}(A \otimes B)=B \dot{\otimes} A=(B \otimes A) K_{m q} \\
& A \otimes B I_{n} \dot{\otimes} I_{p}=A \dot{\otimes} B \\
& A \dot{\otimes} B C \dot{\otimes} D=A D \otimes B C \\
& A \dot{\otimes} B K_{m q}=A \otimes B \\
& I_{m} \dot{\otimes} I_{q}=K_{m q} \\
& I_{n} \ddot{\otimes} I_{p}=\sum_{i=1}^{n} \sum_{j=1}^{p} H_{i j} \otimes H_{i j} \\
& A \ddot{\otimes} B=I \otimes A^{\prime}\left(\sum_{i=1}^{n} \sum_{j=1}^{q} L_{i j} \otimes L_{i j}\right) B^{\prime} \otimes I \\
& A \ddot{\otimes} B=\sum_{k=1}^{m} \sum_{l=1}^{p} A R_{k l} \otimes R_{k l} B \\
& =\sum_{u=1}^{n} \sum_{v=1}^{q} L_{u v} B^{\prime} \otimes A^{\prime} L_{u v} \\
& K_{m n}=\sum_{s=1}^{m} \sum_{t=1}^{n} R_{s t} \otimes R_{s t}^{\prime} \\
& A \dot{\otimes} B=K_{n p}(B \otimes A)=(A \otimes B) K_{m q} \\
& C \otimes D A \dot{\otimes} B=C A \dot{\otimes} D B \\
& A \dot{\otimes} B E \otimes F=A F \dot{\otimes} B E \\
& K_{n p} A \dot{\otimes} B=B \otimes A \\
& I_{n} \dot{\otimes} I_{p}=\sum_{i=1}^{n} \sum_{j=1}^{p} H_{i j} \otimes H_{i j}^{\prime}=K_{n p} \\
& \sum_{i=1}^{n} \sum_{j=1}^{p} H_{i j} \ddot{\otimes} H_{i j}^{\prime}=\sum_{i=1}^{n} \sum_{j=1}^{p} H_{i j} \otimes H_{i j}^{\prime} \\
& I_{n} \otimes I_{p}=\sum_{i=1}^{n} \sum_{j=1}^{p} H_{i j} \ddot{\otimes} H_{i j} \\
& A \ddot{\otimes} B=A \otimes I\left(\sum_{i=1}^{m} \sum_{j=1}^{p} R_{i j} \otimes R_{i j}\right) I \otimes B \\
& A \ddot{\otimes} B K_{m n}^{\prime}=A \ddot{\otimes} B^{\prime} \\
& A \ddot{\otimes} B C \otimes D=A \ddot{\otimes} C^{\prime} B D \\
& A \ddot{\otimes} B C \dot{\otimes} D=A \ddot{\otimes} D^{\prime} B^{\prime} C \\
& A \ddot{\otimes} B C \ddot{\otimes} D=\operatorname{tr}\left(B C^{\prime}\right) A \ddot{\otimes} D \\
& (A \dot{\otimes} B)^{-1}=B^{-1} \dot{\otimes} A^{-1} \\
& \operatorname{det}(A \dot{\otimes} B)=\operatorname{det}(A \otimes B) \\
& =(\operatorname{det} A)^{p}(\operatorname{det} B)^{n} \\
& \operatorname{tr}(A \dot{\otimes} B)=\operatorname{tr}(A B), \text { si } n=p=m=q \\
& \operatorname{tr}(A \otimes B)=\operatorname{tr}(A) \operatorname{tr}(B) \text {, si } n=m, p=q \\
& \text { Si } A \text { y } B \text { son ortogonales, } A \otimes B \text { es ortogonal } \\
& \operatorname{tr}(A \ddot{\otimes} B)=\operatorname{tr}\left(A B^{\prime}\right) \text {, si } n=p \\
& A \ddot{\otimes} B \text { es singular y } \operatorname{rang}(A \ddot{\otimes} B)=1 \\
& \text { Si } A>0 \text { y } B>0 \text {, entonces } A \otimes B>0
\end{aligned}
$$

\subsection{Propiedades}

Usando esta nomenclatura podemos determinar algunas propiedades concernientes a estos productos de Kronecker. 
1. $\quad \underset{n \times m}{A} \underset{\substack{p \times q \\ p r \times q s}}{\dot{\otimes}}(\underset{r \times s}{C}))_{\alpha \beta}=(A \dot{\otimes}(B \otimes C))_{p r(i-1)+k^{\prime}, m\left(j^{\prime}-1\right)+l}=a_{i l}(B \otimes C)_{r(k-1)+x, s(j-1)+y}=$ $a_{i l} b_{k j} c_{x y}$ de lo que deducimos $(A \dot{\otimes}(B \otimes C))_{i j k y x l}=a_{i l} b_{k j} c_{x y}$, o sea $(A \dot{\otimes}(B \otimes$ $C))_{i j k l x y}=a_{i y} b_{k j} c_{x l}$. La matriz es de tamaño $n p r \times m q s, \alpha=\operatorname{pr}(i-1)+r(k-1)+x$, $\beta=m s(j-1)+m(y-1)+l$.

2. $\quad((A \dot{\otimes} B) \otimes C)_{\alpha \beta}=((A \dot{\otimes} B) \otimes C)_{r(a-1)+x, s(b-1)+y}=(A \dot{\otimes} B)_{p(i-1)+k, m(j-1)+l} c_{x y}=$ $a_{i l} b_{k j} c_{x y}$

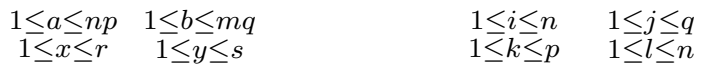

o sea $((A \dot{\otimes} B) \otimes C))_{i j k l x y}=a_{i l} b_{k j} c_{x y}$ y deducimos $(A \dot{\otimes} B) \otimes C \neq A \dot{\otimes}(B \otimes C)$. La matriz es de tamaño $n p r \times m q s, \alpha=\operatorname{pr}(i-1)+r(k-1)+x, \beta=m s(j-1)+s(l-1)+y$.

3. $\quad((A \otimes B) \dot{\otimes} C)_{i j k l x y}=a_{i l} b_{k y} c_{x j}, \quad n p r \times m q s, \alpha=r p(i-1)+r(k-1)+x ; \quad \beta=$ $m q(j-1)+q(l-1)+y$

4. $(A \otimes(B \dot{\otimes} C))_{i j k l x y}=a_{i j} b_{k y} c_{x l}, \quad n p r \times m q s, \alpha=r p(i-1)+r(k-1)+x ; \quad \beta=$ $m q(j-1)+q(l-1)+y$

5. $(A \dot{\otimes}(B \dot{\otimes} C))_{i j k l x y}=a_{i y} b_{k l} c_{x j}, \quad n p r \times m q s, \alpha=r p(i-1)+r(k-1)+x ; \quad \beta=$ $m q(j-1)+q(l-1)+y$

6. $\quad(A \otimes(B \ddot{\otimes} C))_{i j k l x y}=a_{i j} b_{k x} c_{l y}, \quad n p q \times m r s, \alpha=p q(i-1)+q(k-1)+l ; \quad \beta=$ $r s(j-1)+s(x-1)+y$

7. $((A \ddot{\otimes} B) \otimes C)_{i j k l x y}=a_{i k} b_{j l} c_{x y}, \quad n m r \times p q s, \alpha=r m(i-1)+r(k-1)+x ; \beta=$ $s q(j-1)+s(l-1)+y$

8. $\quad((A \ddot{\otimes} B) \dot{\otimes} C)_{i j k l x y}=a_{i k} b_{l y} c_{x j}, \quad n r m \times s p q, \alpha=r m(i-1)+r(k-1)+x ; \quad \beta=$ $p q(j-1)+q(l-1)+y$

9. $(A \dot{\otimes}(B \ddot{\otimes} C))_{i j k l x y}=a_{i y} b_{k x} c_{j l}, \quad n p q \times r m s, \alpha=p q(i-1)+q(k-1)+x ; \quad \beta=$ $m s(j-1)+m(l-1)+y$

10. $A \dot{\otimes}(B \dot{\otimes} C)=(A \dot{\otimes} B) \dot{\otimes} C$.

11. $A \ddot{\otimes}(B \ddot{\otimes} C)=(A \ddot{\otimes} B) \ddot{\otimes} C$.

12. $(A \ddot{\otimes} B) \dot{\otimes} C) \neq A \ddot{\otimes}(B \dot{\otimes} C)$.

Observación $\quad$ Recordemos que $(A \otimes B) \ddot{\otimes} C=\left[A^{\prime} \otimes B^{\prime}\right]\left[C^{\prime}\right]^{\prime}$ matriz $n p m q \times$ rs y

$$
((A \otimes B) \ddot{\otimes} C)_{m q\left(i^{\prime}-1\right)+k^{\prime}, s(x-1)+y}=(A \otimes B)_{p(i-1)+k, q(j-1)+l} c_{x y}=a_{i j} b_{k l} c_{x y},
$$

donde $1 \leq i^{\prime} \leq n p, 1 \leq k^{\prime} \leq m q, 1 \leq x \leq r, 1 \leq y \leq s, 1 \leq i \leq n, 1 \leq k \leq p, 1 \leq j \leq m$, $1 \leq q \leq l$, i.e.

$$
((A \otimes B) \ddot{\otimes} C)_{m q p(i-1)+m q(k-1)+q(j-1)+l, s(x-1)+y}=a_{i j} b_{k l} c_{x y} .
$$


De igual manera

$$
\begin{aligned}
& (A \ddot{\otimes}(B \otimes C))_{m(i-1)+k, q r s(j-1)+q s(x-1)+s(l-1)+y}=a_{i k} b_{j l} c_{x y} \\
& (A \ddot{\otimes}(B \dot{\otimes} C))_{m(i-1)+k, q r s(j-1)+q s(x-1)+q(y-1)+l}=a_{i k} b_{j l} c_{x y} .
\end{aligned}
$$

Observemos que no podemos escribir los productos $A \ddot{\otimes}(B \otimes C)$ y $(A \otimes B) \ddot{\otimes} C$ como el producto de tres matrices no triviales (vectores o constantes) $A^{*} \otimes B^{*} \otimes C^{*}$.

\subsection{Algunos resultados importantes}

Consideremos una matriz $A_{n \times m}$ y $\mu_{p \times 1}, \rho_{q \times 1}$ vectores, entonces

- $(A \dot{\otimes} \mu) \otimes \rho=A \otimes \mu \rho^{\prime}$

- $\left(\mu^{\prime} \dot{\otimes} A\right) \otimes \rho=A \otimes \rho \mu^{\prime}$

- $(\mu \otimes A) \otimes \rho^{\prime}=\mu \rho^{\prime} \dot{\otimes} A$

- $\mu^{\prime} \otimes(A \ddot{\otimes} \rho)=A \ddot{\otimes} \mu \rho^{\prime}$

- $\mu^{\prime} \otimes(A \otimes \rho)=A \dot{\otimes} \mu \rho^{\prime}$

- $\left(\mu^{\prime} \ddot{\otimes} A\right) \otimes \rho=\mu \rho^{\prime} \ddot{\otimes} A$.

\section{Matrices de Kronecker}

La manera de multipicar matrices usando productos de Kronecker, nos sugiere la idea de una clase particular de matrices que llamaremos matrices de Kronecker.

Definición 4 Una matriz $C$ es una matriz de Kronecker si existen matrices $A$ y $B$ no triviales tales que $C=A \otimes B o C=A \dot{\otimes} B o C=A \ddot{\otimes} B$.

Vamos estudiar aquí algunos casos particularmente interesantes. Sea $M=\left(M^{1}, \ldots, M^{t}\right)$ una matriz $p \times t q \operatorname{com} M_{p \times q}^{i}, i=1, \ldots, t$. Sea $A_{n \times m}$ una matriz, entonces $A \otimes M=\left(a_{i j} M\right)$ es una matriz $n p \times m t q$.

\subsection{Estudio del caso $\otimes$}

1. La entrada $(A \otimes M)_{i j k \rho}=a_{i j} m_{k \rho}$, donde la entada $k \rho$ de $M$ se identifica por $m_{k \rho}=$ $m_{k l}^{s}$, con $\rho=(s-1) q+l, 1 \leq s \leq t, 1 \leq l \leq q$, o sea la entrada $k \rho$ de $M$ se identifica con la entrada $k l$ de bloque $s$ de $M, 1 \leq i \leq n, 1 \leq j \leq m, 1 \leq k \leq p, 1 \leq \rho \leq t q$.

Así $(A \otimes M)_{\alpha ; \beta=p(i-1)+k ; t q(j-1)+\rho=p(i-1)+k ; t q(j-1)+q(s-1)+l}$.

Se puede estar tentado a considerar $(A \otimes M)_{i j k s 1 l}$ para identificar la representación anterior de $A \otimes M$, pero no debemos olvidar que $A \otimes M$ no es un producto de tres matrices. 
Si $A=a$ es $n \times 1,(a \otimes M)_{i 1 k \rho}=a_{i} m_{k l}^{s}, n p \times t q$. Sin embargo, sin ser $a \otimes M$ un producto $A^{*} \otimes M^{*}, n p \times t q$ se puede identificar la entrada $i s k l$ de $a \otimes M$ de la siguiente manera $(a \otimes M)_{p(i-1)+k ; q(s-1)+l}=(a \otimes M)_{i 1 k \rho}=a_{i} m_{k l}^{s}$. No es correcto escribir $(a \otimes M)_{i s k l}=a_{i} m_{k l}^{s}$ ya que $a \otimes M$ no es de la forma $A^{*} \otimes M^{*}$ para $A_{n \times m}^{*}$, $M_{p \times q}^{*}$. Sin embargo se puede determinar la entrada $i s k l$ de $a \otimes M$, la cual es $a_{i} m_{k l}^{s}$, pues

$$
a \otimes M=\left(\begin{array}{ccc}
a_{1} M^{1} & \cdots & a_{1} M^{t} \\
\vdots & \ddots & \vdots \\
a_{n} M^{1} & \cdots & a_{n} M^{t}
\end{array}\right)
$$

que es $n p \times t q$, con $1 \leq i \leq n, 1 \leq s \leq t, 1 \leq k \leq p, 1 \leq l \leq q$, por lo que $(a \otimes M)_{i s k l}=a_{i} m_{k l}^{s}$. Sabemos que la notación $(a \otimes M)_{i s k l}$ no es correcta pero en algunas ocasiones es muy útil. Lo correcto es $(a \otimes M)_{i 1 k \rho}$, donde $\rho=(s-1) q+l$.

Si $t=1, \rho=l$, i.e. $(A \otimes M)_{i j k l}=a_{i j} m_{k l}^{1}, n p \times m q$ y además $(a \otimes M)_{i 1 k l}=a_{i} m_{k l}$, $n p \times q$.

2. La matriz $M \otimes A$ es $n p \times m t q$ por lo que $(M \otimes A)_{i \rho k l}=m_{i \rho} a_{k l}=m_{i j}^{s} a_{k l}$, con $\rho=(s-1) q+j, 1 \leq s \leq t, 1 \leq i \leq p, 1 \leq \rho \leq q t, 1 \leq j \leq q, 1 \leq k \leq n, 1 \leq l \leq m$.

Si $t=1, \rho=j,(M \otimes A)_{i j k l}=m_{i j}^{1} a_{k l}, n p \times m q$.

Si $A=a_{n \times 1}, l=1,(M \otimes a)_{i \rho k 1}=a_{k} m_{i \rho}=a_{k} m_{i j}^{s}=(M \otimes a)_{i s k j}$.

Si $t=1,(M \otimes a)_{i j k 1}=m_{i j} a_{k}$ lo que indica el peligro de esta notación pues se está tentado a escribir que si $t=1,(M \otimes a)_{i j k 1}=(M \otimes a)_{i 1 k j}$ que no tiene sentido.

3. La matriz $\left(A^{\prime} \otimes M^{\prime}\right)$ es $t m q \times n p$, i.e. $\left(A^{\prime} \otimes M^{\prime}\right)_{i j \rho l}=m_{\rho l}^{\prime} a_{i j}^{\prime}=m_{l k}^{s} a_{j i}$, con $\rho=$ $(s-1) q+k, 1 \leq s \leq t, 1 \leq k \leq q, 1 \leq j \leq n, 1 \leq i \leq m$.

Si $t=1, \rho=k,\left(A^{\prime} \otimes M^{\prime}\right)_{i j k l}=m_{l k}^{1} a_{j i}, m q \times n p$.

$\left(a^{\prime} \otimes M^{\prime}\right)_{1 j \rho l}=a_{j} m_{l k}^{s}=\left(a^{\prime} \otimes M^{\prime}\right)_{s j k l}$ y si $t=1,\left(a^{\prime} \otimes M^{\prime}\right)_{1 j k 1}=m_{l k} a_{j}$.

4. La matriz $\left(M^{\prime} \otimes A^{\prime}\right)$ es $m t q \times n p$ i.e. $\left(M^{\prime} \otimes A^{\prime}\right)_{\rho j k l}=m_{j \rho} a_{l k}=m_{j i}^{s} a_{l k}$, con $\rho=$ $(s-1) q+i, 1 \leq s \leq t, 1 \leq i \leq q, 1 \leq j \leq p, 1 \leq l \leq n, 1 \leq k \leq m$.

Si $t=1, \rho=i,\left(M^{\prime} \otimes A^{\prime}\right)_{i j k l}=m_{i j}^{1} a_{l k}, m q \times n p$.

$\left(M^{\prime} \otimes a^{\prime}\right)_{\rho j 1 l}=a_{l} m_{j i}^{s}=\left(M^{\prime} \otimes a^{\prime}\right)_{s j i l}$ y si $t=1,\left(M^{\prime} \otimes a^{\prime}\right)_{i j 1 l}=m_{i j} a_{l}$.

Observamos que la notación $\left(M^{\prime} \otimes a^{\prime}\right)_{\text {sjil }}$ se presta a confusión pues en el caso $t=1$, se tiene $s=1$ i.e. se está tentado a escribir $\left(M^{\prime} \otimes a^{\prime}\right)_{1 j i l}=\left(M^{\prime} \otimes a^{\prime}\right)_{i j 1 l}$, lo que no tiene sentido. 


\section{2. $\quad$ Estudio del caso $\dot{\theta}$}

1. La entrada $(A \dot{\otimes} M)_{i \rho k l}=a_{i l} m_{k \rho}=a_{i l} m_{k j}^{s}$, donde $\rho=(s-1) q+j, 1 \leq s \leq t$, $1 \leq j \leq q, 1 \leq i \leq n, 1 \leq l \leq m, 1 \leq k \leq p$.

Así $(A \dot{\otimes} M)_{p(i-1)+k ; m(\rho-1)+l=p(i-1)+k ; m q(s-1)+m(j-1)+l}, n p \times m t q$.

Si $t=1, \rho=j$, i.e. $(A \dot{\otimes} M)_{i j k l}=a_{i l} m_{k j}^{1}, n p \times m q$.

$(a \dot{\otimes} M)_{i \rho k 1}=a_{i} m_{k j}^{s}$, y si $t=1,(a \dot{\otimes} M)_{i j k 1}=a_{i} m_{k j} n p \times q$.

Observemos que $(a \dot{\otimes} M)$ es de tamaño $n p \times t q$ y se podría buscar la entrada $i s k j$ por $(a \dot{\otimes} M)_{i s k j}=a_{i} m_{k j}^{s}$ y si $t=1$ se escribe $(a \dot{\otimes} M)_{i 1 k j}=(a \dot{\otimes} M)_{i j k 1}$ que no tiene sentido.

2. $(M \dot{\otimes} A)_{i j k \rho}=m_{i \rho} a_{k j}=m_{i l}^{s} a_{k j}$, con $\rho=(s-1) q+j, 1 \leq s \leq t, 1 \leq l \leq q, 1 \leq i \leq p$, $1 \leq k \leq n, 1 \leq j \leq m, n p \times m t q$.

Así $(M \dot{\otimes} A)_{n(i-1)+k ; t q(j-1)+\rho=n(i-1)+k ; t q(j-1)+q(s-1)+l}$.

Si $t=1, \rho=l,(M \dot{\otimes} A)_{i j k l}=m_{i l}^{1} a_{k j}, n p \times m q$.

$(M \dot{\otimes} a)_{i 1 k \rho}=a_{k} m_{i \rho}=a_{k} m_{i l}^{s}$. Si $t=1,(M \dot{\otimes} a)_{i 1 k l}=a_{k} m_{i l}$.

La entrada $i s k l$ de la matriz $(M \dot{\otimes} a) n p \times t q$ es $(M \dot{\otimes} a)_{i s k l}=a_{k} m_{i l}^{s}=(M \dot{\otimes} a)_{i 1 k \rho}$ y si $t=1$ las notaciones coinciden.

3. La matriz $\left(A^{\prime} \dot{\otimes} M^{\prime}\right)$ es $m t q \times n p$ por lo que $\left(A^{\prime} \dot{\otimes} M^{\prime}\right)_{i j \rho l}=m_{j \rho} a_{l i}=m_{j k}^{s} a_{l i}$, con $\rho=(s-1) q+k, 1 \leq k \leq q, 1 \leq l \leq n, 1 \leq i \leq m, 1 \leq j \leq p$.

Así $\left(A^{\prime} \dot{\otimes} M^{\prime}\right)_{t q(i-1)+\rho ; n(j-1)+l=t q(i-1)+q(s-1)+k ; n(j-1)+l}$.

Si $t=1, \rho=k,\left(A^{\prime} \dot{\otimes} M^{\prime}\right)_{i j k l}=m_{j k} a_{l i}, m q \times n p$.

$\left(a^{\prime} \dot{\otimes} M^{\prime}\right)_{1 j \rho l}=a_{l} m_{j k}^{s}$ y si $t=1,\left(a^{\prime} \dot{\otimes} M^{\prime}\right)_{1 j k l}=a_{l} m_{j k}$.

La entrada $s j k l$ de la matriz $\left(a^{\prime} \dot{\otimes} M^{\prime}\right) t q \times n p$ es $\left(a^{\prime} \dot{\otimes} M^{\prime}\right)_{s j k l}=a_{l} m_{j k}^{s}$ y escribimos $\left(a^{\prime} \dot{\otimes} M^{\prime}\right)_{1 j \rho l}=\left(a^{\prime} \dot{\otimes} M^{\prime}\right)_{s j k l}$ y si $t=1$ las notaciones coinciden.

4. La matriz $\left(M^{\prime} \dot{\otimes} A^{\prime}\right)$ es $m t q \times n p$ por lo que $\left(M^{\prime} \dot{\otimes} A^{\prime}\right)_{\rho j k l}=m_{l i}^{s} a_{j k}$, con $\rho=(s-$ 1) $q+i, 1 \leq i \leq q, 1 \leq j \leq n, 1 \leq k \leq m, 1 \leq l \leq p$.

Así $\left(A^{\prime} \dot{\otimes} M^{\prime}\right)_{\rho j k l=m q(s-1)+m(i-1)+k ; p(j-1)+l}$.

Si $t=1, \rho=i,\left(M^{\prime} \dot{\otimes} A^{\prime}\right)_{i j k l}=m_{l i} a_{j k}, m q \times n p$.

$\left(M^{\prime} \dot{\otimes} a^{\prime}\right)_{\rho j 1 l}=a_{j} m_{l i}^{s}=\left(M^{\prime} \dot{\otimes} a^{\prime}\right)_{s j i l}$ y si $t=1,\left(M^{\prime} \dot{\otimes} a^{\prime}\right)_{i j 1 l}=\left(M^{\prime} \dot{\otimes} a^{\prime}\right)_{1 j i l}$ y las notaciones coinciden. 


\subsection{Estudio del caso $\ddot{\otimes}$}

1. La entrada $(A \ddot{\otimes} M)_{i j k \rho}=a_{i k} m_{j l}^{s}$, donde $\rho=(s-1) q+l, 1 \leq l \leq q, 1 \leq j \leq p$, $1 \leq i \leq n, 1 \leq k \leq m, 1 \leq s \leq t$. Así $(A \ddot{\otimes} M)_{m(i-1)+k ; t q(j-1)+q(s-1)+l}, n m \times t p q$.

Si $t=1, \rho=l$, i.e. $(A \ddot{\otimes} M)_{i j k l}=a_{i k} m_{j l}^{1}, n m \times p q$.

$(a \ddot{\otimes} M)_{i j 1 \rho}=a_{i} m_{j l}^{s}$, y si $t=1,(a \ddot{\otimes} M)_{i j 1 l}=a_{i} m_{j l} n \times p q$.

2. $(M \ddot{\otimes} A)_{i j \rho l}=m_{i \rho} a_{j l}=m_{i k}^{s} a_{j l}$, con $\rho=(s-1) q+k, 1 \leq s \leq t, 1 \leq k \leq q, 1 \leq i \leq p$, $1 \leq j \leq n, 1 \leq l \leq m, t p q \times n m$.

Si $t=1, \rho=k,(M \ddot{\otimes} A)_{i j k l}=m_{i k}^{1} a_{j l}, p q \times n m$.

$(M \ddot{\otimes} a)_{i j \rho 1}=a_{j} m_{i k}^{s}$. Si $t=1,(M \ddot{\otimes} a)_{i j k 1}=a_{j} m_{i k}$.

$(M \ddot{\otimes} a)_{i j \rho 1}=a_{j} m_{i k}^{s}$ y si $t=1,(M \ddot{\otimes} a)_{i j k 1}=a_{j} m_{i k}$.

3. La matriz $\left(A^{\prime} \ddot{\otimes} M^{\prime}\right)$ es $n m \times t p q$ por lo que $\left(A^{\prime} \ddot{\otimes} M^{\prime}\right)_{i \rho k l}=m_{l \rho} a_{k i}=m_{l j}^{s} a_{k i}$, con $\rho=(s-1) q+j, 1 \leq s \leq t, 1 \leq j \leq q, 1 \leq k \leq n, 1 \leq i \leq m, 1 \leq l \leq p$.

Si $t=1, \rho=j,\left(A^{\prime} \ddot{\otimes} M^{\prime}\right)_{i j k l}=m_{l j} a_{k i}, n m \times p q$.

$\left(a^{\prime} \ddot{\otimes} M^{\prime}\right)_{1 \rho k l}=a_{k} m_{l j}^{s}$ y si $t=1,\left(a^{\prime} \ddot{\otimes} M^{\prime}\right)_{1 j k l}=a_{k} m_{l j}$.

4. La matriz $\left(M^{\prime} \dot{\otimes} A^{\prime}\right)$ es $t p q \times n m$ por lo que $\left(M^{\prime} \dot{\otimes} A^{\prime}\right)_{\rho j k l}=m_{k i}^{s} a_{l j}$, con $\rho=(s-$ 1) $q+i, 1 \leq i \leq q, 1 \leq s \leq t, 1 \leq k \leq p, 1 \leq l \leq n, 1 \leq j \leq m$.

Si $t=1, \rho=i,\left(M^{\prime} \ddot{\otimes} A^{\prime}\right)_{i j k l}=m_{k i} a_{l j}, p q \times n m$.

$\left(M^{\prime} \ddot{\otimes} a^{\prime}\right)_{\rho 1 k l}=a_{l} m_{k i}^{s}=\left(M^{\prime} \ddot{\otimes} a^{\prime}\right)_{s j i l}$ y si $t=1\left(M^{\prime} \ddot{\otimes} a^{\prime}\right)_{i 1 k l}=a_{l} m_{k i}$.

\section{Cálculo de momentos de orden 3}

Consideremos $x_{n \times 1}, y_{m \times 1}, z_{p \times 1}, u_{q \times 1}$ vectores aleatorios y consideremos $E\left(y^{\prime} \otimes z \otimes u^{\prime}\right)=$ $E\left(y^{\prime} \otimes z u^{\prime}\right)=E\left(y_{1} z u^{\prime}, \ldots, y_{m} z u^{\prime}\right)=\left(M^{1}, \ldots, M^{m}\right)=M_{y u}^{\cdot z}$, con $M^{i}=E\left(y_{i} z u^{\prime}\right)_{p \times q}$, de dimensión $p \times m q$. Recordemos que $\left(M_{y u}^{\cdot z}\right)_{1 j k l}=m_{k l}^{j}=E\left(y_{j}\left(z u^{\prime}\right)_{k l}\right) . E\left(y \otimes z^{\prime} \otimes u\right)=$ $E\left(y z^{\prime} \otimes u\right)=\left(\begin{array}{c}y z^{\prime} u_{1} \\ \vdots \\ y z^{\prime} u_{q}\end{array}\right)=\left(\begin{array}{c}N^{1} \\ \vdots \\ N^{q}\end{array}\right)=M_{y u}^{\cdot z}$, con $N^{i}=E\left(u_{i} y z^{\prime}\right)_{m \times p}$ de dimensión $m q \times p$. Ahora

$$
\begin{aligned}
& \left.\begin{array}{ll}
E\left(\left(y \otimes z^{\prime} \otimes u\right)^{\prime}\right) & =M_{y u}^{\cdot z} \\
E\left(y \otimes z^{\prime} \otimes u\right) & =M_{\cdot z}^{y u}
\end{array}\right\} \Rightarrow\left(M_{y u}^{\cdot z}\right)^{\prime}=M_{\cdot z}^{y u} \\
& E\left(x^{\prime} \otimes z \otimes u\right)=E\left(z \otimes x^{\prime} \otimes u\right)=\left(M_{\cdot x}^{x u}\right)^{\prime} \\
& E\left(x \otimes z \otimes u^{\prime}\right)=E\left(x \otimes z u^{\prime}\right)=E\left(\begin{array}{c}
x_{1} z u^{\prime} \\
\vdots \\
x_{n} z u^{\prime}
\end{array}\right)=\left(\begin{array}{c}
M^{1} \\
\vdots \\
M^{n}
\end{array}\right)=\left(M_{\cdot u}^{x z}\right)^{\prime} .
\end{aligned}
$$


En resumen

1. $E\left(x^{\prime} \otimes z \otimes u^{\prime}\right)=M_{x u}^{\cdot z}, p \times n q$

2. $E\left(x \otimes z^{\prime} \otimes u\right)=M_{\cdot z}^{x u}, n q \times p$

3. $\quad E\left(x \otimes z \otimes u^{\prime}\right)=E\left(x \otimes u^{\prime} \otimes z\right)=M_{\cdot u}^{x z}, n p \times q$

4. $\quad E\left(x^{\prime} \otimes z^{\prime} \otimes u\right)=E\left(x^{\prime} \otimes u \otimes z^{\prime}\right)=M_{x z}^{\cdot u}, q \times n p$

5. $\quad E\left(x \otimes z^{\prime} \otimes u^{\prime}\right)=E\left(z^{\prime} \otimes x \otimes u^{\prime}\right)=M_{z u}^{\cdot x}, \quad n \times p q$

6. $\quad E\left(x^{\prime} \otimes z \otimes u\right)=E\left(z \otimes x^{\prime} \otimes u\right)=M_{\cdot x}^{z u}, p q \times n$.

Observemos que si $x=z=u, E\left(x \otimes x^{\prime} \otimes x\right)=M_{x x}^{x}=M_{3}^{\prime}, n^{2} \times n$ y $\quad E\left(x^{\prime} \otimes x \otimes x\right)=$ $M_{x x}^{\cdot x}=M_{3}, n \times n^{2}$.

a) Consideremos la expresión siguiente

$$
\begin{aligned}
& (x-\mu) \otimes(y-\nu)(z-\rho)^{\prime}=(x-\mu) \otimes\left(y z^{\prime}-\nu z^{\prime}-y \rho^{\prime}+\nu \rho^{\prime}\right)= \\
& x \otimes y z^{\prime}-x \otimes \nu z^{\prime}-x \otimes y \rho^{\prime}+x \otimes \nu \rho^{\prime}-\mu \otimes y z^{\prime}+\mu \otimes \nu z^{\prime}+\mu \otimes y \rho^{\prime}-\mu \otimes \nu \rho^{\prime}= \\
& x \otimes y \otimes z^{\prime}-x z^{\prime} \dot{\otimes}-x y^{\prime} \ddot{\otimes} \rho^{\prime}+x \otimes \nu \rho^{\prime}-\mu \otimes y z^{\prime}+\mu \otimes \nu z^{\prime}+\mu \otimes y \rho^{\prime}-\mu \otimes \nu \rho^{\prime} . \\
& E\left((x-\mu) \otimes(y-\nu)(z-\rho)^{\prime}\right)=M_{x y}^{\cdot z}-M_{x z} \dot{\otimes} \nu-M_{y x}^{\prime} \ddot{\otimes} \rho^{\prime}-\mu \otimes M_{y z}+2 \mu \otimes \nu \rho^{\prime} .
\end{aligned}
$$

Si $x=y=z, E\left((x-\mu) \otimes(x-\mu)(x-\mu)^{\prime}\right)=M_{3}-M_{2} \dot{\otimes} \mu-M_{2} \ddot{\otimes} \mu^{\prime}-\mu \otimes M_{2}+2 \mu \otimes \mu \mu^{\prime}$, donde $M_{2}=M_{x x}=\Sigma+\mu \mu^{\prime}$ i.e. $E\left((x-\mu) \otimes(x-\mu)(x-\mu)^{\prime}\right)=M_{3}-\Sigma \dot{\otimes} \mu-\Sigma \ddot{\otimes} \mu^{\prime}-$ $\mu \otimes \Sigma-\mu \otimes \mu \mu^{\prime}$.

b) Consideremos la expresión siguiente

$$
\begin{aligned}
& (x-\mu) \ddot{\otimes}(y-\nu)(z-\rho)^{\prime}=(x-\mu) 1^{\prime} \ddot{\otimes}(y-\nu)(z-\rho)^{\prime}=(x-\mu)(y-\nu)^{\prime} \otimes 1(z-\rho)^{\prime}= \\
& x \ddot{\otimes} y z^{\prime}-x \ddot{\otimes} \nu z^{\prime}-x \ddot{\otimes} y \rho^{\prime}+x \ddot{\otimes} \nu \rho^{\prime}-\mu \ddot{\otimes} y z^{\prime}+\mu \ddot{\otimes} \nu z^{\prime}+\mu \ddot{\otimes} y \rho^{\prime}-\mu \ddot{\otimes} \nu \rho^{\prime} . \\
& E\left((x-\mu) \ddot{\otimes}(y-\nu)(z-\rho)^{\prime}\right)=M_{y z}^{\cdot x}-M_{z x}^{\prime} \dot{\otimes} \nu^{\prime}-M_{y x}^{\prime} \otimes \rho^{\prime}-\mu \ddot{\otimes} M_{x z}+2 \mu \ddot{\otimes} \nu \rho^{\prime} .
\end{aligned}
$$

\section{Momentos de orden 4}

Consideremos $x_{n \times 1}, y_{m \times 1}, z_{p \times 1}, u_{q \times 1}$ vectores aleatorios. La esperanza matemática $E\left(x y^{\prime} \otimes z u^{\prime}\right)_{i j k l}=E\left(x_{i} y_{j} z_{k} u_{l}\right)=M_{i j k l}=\left(M_{y u}^{x z}\right)_{i j k l}$, donde $M_{y u}^{x z}$ es la matriz $n p \times m q$ de momentos de orden 4 , de las variables $x, y, z, u$.

La expresión $E\left((x-\mu)(y-\nu)^{\prime} \otimes(z-\xi)(u-\theta)^{\prime}\right)=\bar{M}_{y u}^{x z}$ matriz de momentos centrados de orden 4 , de las variables $x, y, z, u$. Así

$$
\begin{aligned}
& E\left(x y^{\prime} \otimes z u^{\prime}-x y^{\prime} \otimes z \theta^{\prime}-x y^{\prime} \otimes \xi u^{\prime}+x y^{\prime} \otimes \xi \theta^{\prime}-\mu y^{\prime} \otimes z u^{\prime}+\mu y^{\prime} \otimes z \theta^{\prime}+\mu y^{\prime} \otimes \xi u^{\prime}-x y^{\prime} \otimes \xi \theta^{\prime}\right. \\
& \left.-x \nu^{\prime} \otimes z u^{\prime}+x \nu^{\prime} \otimes z \theta^{\prime}+x \nu \otimes z u^{\prime}-x \nu^{\prime} \otimes \xi \theta^{\prime}+\mu \nu^{\prime} \otimes z u^{\prime}-\mu \nu^{\prime} \otimes z \theta^{\prime}-\mu \nu^{\prime} \otimes \xi u^{\prime}+\mu \nu^{\prime} \otimes \xi \theta^{\prime}\right) \\
& =M_{y u}^{x z}-\left(M_{x z}^{\cdot y}\right)^{\prime} \otimes \theta^{\prime}-M_{y u}^{\cdot x} \otimes \xi-\mu \otimes M_{y u}^{z}-\nu^{\prime} \otimes\left(M_{x z}^{\cdot}\right)^{\prime}+M_{x y} \otimes \xi \theta^{\prime}+\mu \theta^{\prime} \dot{\otimes} M_{z y} \\
& +M_{x u} \dot{\otimes} \nu^{\prime}+\mu \nu^{\prime} \otimes M_{z u}+\mu \xi^{\prime} \ddot{\otimes} M_{y u}+M_{x z} \ddot{\otimes} \nu \theta^{\prime}-3 \mu \nu^{\prime} \otimes \xi \theta^{\prime} .
\end{aligned}
$$

Observemos que: 
1. $E\left(\mu y^{\prime} \otimes z u^{\prime}\right)=\mu \otimes E\left(y^{\prime} \otimes z \otimes u^{\prime}\right)=\mu \otimes M_{y u}^{\cdot z}$

2. $\quad E\left(x y^{\prime} \otimes \xi u^{\prime}\right)=E\left(x \otimes y^{\prime} \otimes u^{\prime} \otimes \xi\right)=M_{y u}^{\cdot x} \otimes \xi$

3. $\quad E\left(x \nu^{\prime} \otimes z u^{\prime}\right)=E\left(\nu^{\prime} \otimes x \otimes z \otimes u^{\prime}\right)=\nu^{\prime} \otimes M_{\cdot u}^{x z}=\nu^{\prime} \otimes\left(M_{x z}^{\cdot u}\right)^{\prime}$

4. $\quad E\left(x y^{\prime} \otimes z \theta^{\prime}\right)=E\left(x \otimes y^{\prime} \otimes z\right) \otimes \theta^{\prime}=M_{\cdot y}^{x z} \otimes \theta^{\prime}=\left(M_{x z}^{\cdot y}\right)^{\prime} \otimes \theta^{\prime}$

5. $\quad E\left(x \nu^{\prime} \otimes z \theta^{\prime}\right)=E\left(x z^{\prime} \ddot{\otimes} \nu \theta^{\prime}\right)=M_{x z} \ddot{\otimes} \nu \theta^{\prime}$

6. $E\left(x \nu^{\prime} \otimes \xi u^{\prime}\right)=E\left(x \otimes \nu^{\prime} \otimes \xi \otimes u^{\prime}\right)=E\left(\nu^{\prime} \otimes x \otimes u^{\prime} \otimes \xi\right)=\nu^{\prime} \otimes M_{x u} \otimes \xi=M_{x u} \dot{\otimes} \xi \nu^{\prime}$

7. $E\left(x y^{\prime} \otimes \xi \theta^{\prime}\right)=M_{x y} \otimes \xi \theta^{\prime}$

8. $\quad E\left(\mu y^{\prime} \otimes \xi u^{\prime}\right)=E\left(\mu \xi^{\prime} \ddot{\otimes} y u^{\prime}\right)=\mu \xi^{\prime} \ddot{\otimes} M_{y u}$

9. $E\left(\mu \nu^{\prime} \otimes z u^{\prime}\right)=\mu \nu^{\prime} \otimes M_{z u}$.

Si $x=y=z=u$,

$E\left((x-\mu)(x-\mu)^{\prime} \otimes(x-\mu)(x-\mu)^{\prime}\right)=\bar{M}_{4}=M_{4}-M_{3}^{\prime} \otimes \mu^{\prime}-M_{3} \otimes \mu-\mu \otimes M_{3}-\mu^{\prime} \otimes M_{3}^{\prime}+$ $\Sigma \otimes \mu \mu^{\prime}+\mu \mu^{\prime} \otimes \Sigma+\Sigma \dot{\otimes} \mu \mu^{\prime}+\mu \mu^{\prime} \dot{\otimes} \Sigma+\Sigma \ddot{\otimes} \mu \mu^{\prime}+\mu \mu^{\prime} \ddot{\otimes} \Sigma+3 \mu \mu^{\prime} \otimes \mu \mu^{\prime}$

donde $\bar{M}_{4}=\bar{M}_{x x}^{x x}$ y $M_{4}=M_{x x}^{x x}$.

\section{Cálculo de covarianzas}

Sea $Y=\left(Y_{1}, \ldots, Y_{n}\right)$ vectores aleatorios con momentos de orden 4 tales que $E\left(Y_{\alpha}\right)=$ $\mu_{\alpha}, \alpha=1, \ldots, n, E(Y)=\left(\mu_{1}, \ldots, \mu_{n}\right)=\Gamma, \operatorname{cov}\left(Y_{\alpha}, Y_{\beta}\right)=\delta_{\alpha \beta} \Sigma$ y $\bar{M}_{3}, \bar{M}_{4}$ matrices de momentos centrados de orden 3 y 4 respectivamente que no dependen de $\alpha$ y sean $A_{n \times n}$ matriz simétrica, $B_{n \times p}$.

a) Consideremos la forma cuadrática

$$
E\left((Y-\Gamma) A(Y-\Gamma)^{\prime}\right)=\sum_{\alpha=1}^{n} \sum_{\beta=1}^{n} a_{\alpha \beta} E\left(\left(Y_{\alpha}-\mu_{\alpha}\right)\left(Y_{\beta}-\mu_{\beta}\right)^{\prime}\right)=\sum_{\alpha=1}^{n} a_{\alpha \alpha} \Sigma=\operatorname{tr}(A) \Sigma
$$

por lo que $E\left(Y A Y^{\prime}\right)=\Gamma A \Gamma^{\prime}+\operatorname{tr}(A) \Sigma$. Sea $Y B=\left(Y_{1}, \ldots, Y_{n}\right)\left(\begin{array}{c}b_{1}^{\prime} \\ \vdots \\ b_{n}^{\prime}\end{array}\right)=\sum_{\alpha=1}^{n} Y_{\alpha} b_{\alpha}^{\prime}$, $E(Y B)=\Gamma B$. Definimos $X=Y-\Gamma, \Gamma A=\nu=\left(\nu_{1}, \ldots, \nu_{n}\right)$, entonces $Y A Y^{\prime}-$ 
$\Gamma A \Gamma^{\prime}-\operatorname{tr}(A) \Sigma=X A X^{\prime}+\Gamma A X^{\prime}+X A \Gamma^{\prime}-\Sigma \operatorname{tr}(A)$ y $Y B-\Gamma B=X B$. Así

$$
\begin{gathered}
\operatorname{cov}\left(Y A Y^{\prime}, Y B\right)=E\left(\left(\sum_{\alpha=1}^{n} \sum_{\beta=1}^{n} a_{\alpha \beta} X_{\alpha} X_{\beta}^{\prime}+\sum_{\alpha=1}^{n} \nu_{\alpha} X_{\alpha}^{\prime}\right.\right. \\
\left.\left.\quad+\sum_{\alpha=1}^{n} X_{\alpha} \nu_{\alpha}^{\prime}-\Sigma \operatorname{tr}(A)\right)^{\prime} \ddot{\otimes}\left(\sum_{\alpha=1}^{n} X_{\alpha} b_{\alpha}^{\prime}\right)^{\prime}\right) \\
=E\left(\sum_{\alpha \beta \gamma} a_{\alpha \beta} X_{\alpha} X_{\beta}^{\prime} \ddot{\otimes} b_{\gamma} X_{\gamma}^{\prime}+\sum_{\alpha \beta} \nu_{\alpha} X_{\alpha}^{\prime} \ddot{\otimes} b_{\beta} X_{\beta}^{\prime}\right. \\
\left.\quad+\sum_{\alpha \beta} X_{\alpha} \nu_{\alpha}^{\prime} \ddot{\otimes} b_{\beta} X_{\beta}^{\prime}+\operatorname{tr}(A) \Sigma \ddot{\otimes} B^{\prime} X^{\prime}\right) \\
=\sum_{\alpha \beta \gamma} a_{\alpha \beta} E\left(X_{\alpha} X_{\gamma}^{\prime} \otimes X_{\beta}^{\prime}\right) \otimes b_{\gamma}+\sum_{\alpha} \nu_{\alpha} b_{\alpha}^{\prime} \otimes \Sigma \\
\quad+\Sigma \dot{\otimes} \sum_{\alpha} \nu_{\alpha} b_{\alpha}^{\prime} \\
=\bar{M}_{3}^{\prime} \otimes a^{\prime} B+\Gamma A B \otimes \Sigma+\Sigma \dot{\otimes} \Gamma A B,
\end{gathered}
$$

donde $a^{\prime}=\left(a_{11}, \ldots, a_{n n}\right)$ es el vector de tamaño $n \times 1$ con los elementos de la diagonal de $A$.

b) Sean $A_{n \times n}, B_{n \times n}$ matrices simétricas, entonces

$$
\begin{aligned}
& Y A Y^{\prime}-\Gamma A \Gamma^{\prime}-\operatorname{tr}(A) \Sigma=X A X^{\prime}+\Gamma A X^{\prime}+X A \Gamma^{\prime}-\operatorname{tr}(A) \Sigma, \quad \Gamma A=\nu \\
& Y B Y^{\prime}-\Gamma B \Gamma^{\prime}-\operatorname{tr}(B) \Sigma=X B X^{\prime}+\Gamma B X^{\prime}+X B \Gamma^{\prime}-\operatorname{tr}(B) \Sigma, \quad \Gamma B=\rho \\
& \operatorname{cov}\left(Y A Y^{\prime}, Y B Y^{\prime}\right)=E\left(\sum_{\alpha \beta \gamma \delta} a_{\alpha \beta} b_{\gamma \delta} X_{\alpha} X_{\beta}^{\prime} \ddot{\otimes} X_{\gamma} X_{\delta}^{\prime}+\sum_{\alpha \beta \gamma} a_{\alpha \beta} X_{\alpha} X_{\beta}^{\prime} \ddot{\otimes} \rho_{\gamma} X_{\gamma}^{\prime}\right. \\
& +\sum_{\alpha \beta \gamma} a_{\alpha \beta} X_{\alpha} X_{\beta}^{\prime} \ddot{\otimes} X_{\gamma} \rho_{\gamma}^{\prime}-\sum_{\alpha \beta} a_{\alpha \beta} X_{\alpha} X_{\beta}^{\prime} \ddot{\otimes} \Sigma \operatorname{tr}(B) \\
& +\sum_{\alpha \beta \gamma} \nu_{\alpha} X_{\alpha}^{\prime} \ddot{\otimes} b_{\gamma \delta} X_{\gamma} X_{\delta}^{\prime}+\sum_{\alpha \beta} \nu_{\alpha} X_{\alpha}^{\prime} \ddot{\otimes} \rho_{\beta} X_{\beta}^{\prime} \\
& +\sum_{\alpha \beta} \nu_{\alpha} X_{\alpha}^{\prime} \ddot{\otimes} X_{\beta} \rho_{\beta}^{\prime}+\sum_{\alpha \gamma \delta} X_{\alpha} \nu_{\alpha}^{\prime} \ddot{\otimes} b_{\gamma \delta} X_{\gamma} X_{\delta}^{\prime} \\
& +\sum_{\alpha \beta} X_{\alpha} \nu_{\alpha}^{\prime} \ddot{\otimes} \rho_{\beta} X_{\beta}^{\prime}+\sum_{\alpha \beta} X_{\alpha} \nu_{\alpha}^{\prime} \ddot{\otimes} X_{\beta} \rho_{\beta}^{\prime} \\
& \left.-\Sigma \operatorname{tr}(A) \ddot{\otimes} \sum_{\alpha \beta} b_{\alpha \beta} X_{\alpha} X_{\beta}^{\prime}+\operatorname{tr}(A) \operatorname{tr}(B) \Sigma \ddot{\otimes} \Sigma\right) . \\
& -\sum_{\alpha \beta \gamma \delta} a_{\alpha \beta} b_{\gamma \delta} E\left(X_{\alpha} X_{\gamma}^{\prime} \otimes X_{\beta} X_{\delta}^{\prime}\right)=\sum_{\substack{\alpha \neq \beta \\
\alpha=\gamma, \beta=\delta}} a_{\alpha \beta} b_{\alpha \beta} E\left(X_{\alpha} X_{\alpha}^{\prime} \otimes X_{\beta} X_{\beta}^{\prime}\right) \\
& +\sum_{\substack{\alpha \neq \gamma \\
\alpha=\beta, \gamma=\delta}} a_{\alpha \alpha} b_{\gamma \gamma} E\left(X_{\alpha} X_{\gamma}^{\prime} \otimes X_{\alpha} X_{\gamma}^{\prime}\right)+ \\
& \sum_{\substack{\alpha \neq \delta \\
\alpha=\beta, \delta=\gamma}} a_{\alpha \delta} b_{\delta \alpha} E\left(X_{\alpha} X_{\delta}^{\prime} \otimes X_{\delta} X_{\alpha}^{\prime}\right)+\sum_{\alpha} a_{\alpha \alpha} b_{\alpha \alpha} E\left(X_{\alpha} X_{\alpha}^{\prime} \otimes X_{\alpha} X_{\alpha}^{\prime}\right)= \\
& {\left[\operatorname{tr}(A B) \Sigma \otimes \Sigma-a^{\prime} b \Sigma \otimes \Sigma\right]+\left[\operatorname{tr}(A) \operatorname{tr}(B) \Sigma \ddot{\otimes} \Sigma-a^{\prime} b \Sigma \ddot{\otimes} \Sigma\right]+[\operatorname{tr}(A) \operatorname{tr}(B) \Sigma \dot{\otimes} \Sigma-} \\
& \left.a^{\prime} b \Sigma \dot{\otimes} \Sigma\right]+a^{\prime} b \bar{M}_{4}= \\
& a^{\prime} b\left[\bar{M}_{4}-\Sigma \otimes \Sigma-\Sigma \dot{\otimes} \Sigma-\Sigma \ddot{\otimes} \Sigma\right]+\operatorname{tr}(A B)[\Sigma \otimes \Sigma+\Sigma \dot{\otimes} \Sigma]+\operatorname{tr}(A) \operatorname{tr}(B) \Sigma \ddot{\otimes} \Sigma \\
& \text { donde } a=\left(a_{11}, \ldots, a_{n n}\right), b=\left(b_{11}, \ldots, b_{n n}\right) \text {, es decir los elementos diagonales de } A
\end{aligned}
$$


- $\sum_{\alpha \beta \gamma} a_{\alpha \beta} E\left(X_{\alpha} X_{\beta}^{\prime} \ddot{\otimes} \rho_{\gamma} X_{\gamma}^{\prime}\right)=\sum_{\alpha \beta \gamma} a_{\alpha \beta} E\left(X_{\alpha} \rho_{\gamma}^{\prime} \otimes X_{\beta} X_{\gamma}^{\prime}\right)=\sum_{\alpha} a_{\alpha \alpha} \rho_{\alpha}^{\prime} \otimes \bar{M}_{3}^{\prime}=$ $a^{\prime} B \Gamma^{\prime} \otimes \bar{M}_{3}^{\prime}$

- $\sum_{\alpha \beta \gamma} a_{\alpha \beta} E\left(X_{\alpha} X_{\gamma}^{\prime} \otimes X_{\beta}\right) \otimes \rho_{\gamma}^{\prime}=\bar{M}_{3}^{\prime} \otimes a^{\prime} B \Gamma^{\prime}$

- $\sum_{\alpha \gamma \delta} b_{\gamma \delta} E\left(\nu_{\alpha} X_{\gamma}^{\prime} \otimes X_{\alpha} X_{\delta}^{\prime}\right)=\sum_{\alpha} b_{\alpha \alpha} \nu_{\alpha} \otimes \bar{M}_{3}=\Gamma A b \otimes \bar{M}_{3}$

- $\sum_{\alpha \gamma \delta} b_{\gamma \delta} E\left(X_{\alpha} X_{\gamma}^{\prime} \otimes \nu_{\alpha} X_{\delta}^{\prime}\right)=\bar{M}_{3} \otimes \sum_{\alpha} b_{\alpha \alpha} \nu_{\alpha}=\bar{M}_{3} \otimes \Gamma A b$

- $\sum_{\alpha \beta} a_{\alpha \beta} E\left(X_{\alpha} X_{\beta}^{\prime}\right) \ddot{\otimes} \Sigma \operatorname{tr}(B)=\operatorname{tr}(A) \operatorname{tr}(B) \Sigma \ddot{\otimes} \Sigma$

- $\sum_{\alpha \beta} \nu_{\alpha} \rho_{\beta}^{\prime} \otimes E\left(X_{\alpha} X_{\beta}^{\prime}\right)=\Gamma A B \Gamma^{\prime} \otimes \Sigma$

- $\sum_{\alpha \beta} E\left(\nu_{\alpha} X_{\beta}^{\prime} \otimes X_{\alpha} \rho_{\beta}^{\prime}\right)=\sum_{\alpha \beta} \nu_{\alpha} \rho_{\beta}^{\prime} \dot{\otimes} E\left(X_{\alpha} X_{\beta}^{\prime}\right)=\Gamma A B \Gamma^{\prime} \dot{\otimes} \Sigma$

- $\sum_{\alpha \beta} E\left(X_{\alpha} \rho_{\beta}^{\prime} \otimes \nu_{\alpha} X_{\beta}^{\prime}\right)=\sum_{\alpha \beta} E\left(X_{\alpha} X_{\beta}^{\prime}\right) \dot{\otimes} \nu_{\alpha} \rho_{\beta}^{\prime}=\Sigma \dot{\otimes} \Gamma A B \Gamma^{\prime}$

- $\sum_{\alpha \beta} b_{\alpha \beta} E\left(X_{\alpha} X_{\beta}^{\prime}\right) \ddot{\otimes} \Sigma \operatorname{tr}(A)=\operatorname{tr}(A) \operatorname{tr}(B) \Sigma \ddot{\otimes} \Sigma$.

Así tenemos

$$
\begin{aligned}
\operatorname{cov}\left(Y A Y^{\prime}, Y B Y^{\prime}\right)= & a^{\prime} b\left(\bar{M}_{4}-\Sigma \otimes \Sigma-\Sigma \dot{\otimes} \Sigma-\Sigma \ddot{\otimes} \Sigma\right)+\operatorname{tr}(A B)(\Sigma \otimes \Sigma+\Sigma \dot{\otimes} \Sigma)+ \\
& \bar{M}_{3}^{\prime} \otimes a^{\prime} B \Gamma^{\prime}+a^{\prime} B \Gamma^{\prime} \otimes \bar{M}_{3}^{\prime}+\bar{M}_{3} \otimes \Gamma A b+\Gamma A b \otimes \bar{M}_{3}+ \\
& \Gamma A B \Gamma^{\prime} \otimes \Sigma+\Sigma \otimes \Gamma A B \Gamma^{\prime}+\Gamma A B \Gamma^{\prime} \dot{\otimes} \Sigma+\Sigma \dot{\otimes} \Gamma A B \Gamma^{\prime}
\end{aligned}
$$

Nota Si $Y_{\alpha}$ es una variable aleatoria real, $E(Y)=\mu, \operatorname{var}(Y)=\sigma^{2} I$

$$
\operatorname{cov}\left(Y^{\prime} A Y, Y^{\prime} B Y\right)=a^{\prime} b\left(\bar{m}_{4}-3 \sigma^{4}\right)+2 \sigma^{4} \operatorname{tr}(A B)+2 \mu^{\prime} A b \bar{m}_{3}+2 a^{\prime} B \mu \bar{m}_{3}+4 \sigma^{2} \mu^{\prime} A B \mu
$$

c) Si asumimos que $Y$ tiene esperanza $\Gamma$ y covarianza $V \otimes \Sigma$

$$
\begin{aligned}
\operatorname{cov}\left(Y A Y^{\prime}, Y B Y^{\prime}\right)= & a^{\prime} b\left(\bar{M}_{4}-\Sigma \otimes \Sigma-\Sigma \dot{\otimes} \Sigma-\Sigma \ddot{\otimes} \Sigma\right)+\operatorname{tr}(A V B V)(\Sigma \otimes \Sigma+\Sigma \dot{\otimes} \Sigma)+ \\
& \bar{M}_{3}^{\prime} \otimes a^{\prime} B \Gamma^{\prime}+a^{\prime} B \Gamma^{\prime} \otimes \bar{M}_{3}^{\prime}+\bar{M}_{3} \otimes \Gamma A b+\Gamma A b \otimes \bar{M}_{3}+ \\
& \Gamma A V B \Gamma^{\prime} \otimes \Sigma+\Sigma \otimes \Gamma A V B \Gamma^{\prime}+\Gamma A V B \Gamma^{\prime} \dot{\otimes} \Sigma+\Sigma \dot{\otimes} \Gamma A V B \Gamma^{\prime}
\end{aligned}
$$

y si asumimos normalidad i.e. $Y \sim N(\Gamma, V \otimes \Sigma)$, el término en $a^{\prime} b$ es nulo al igual que $\bar{M}_{3}$.

- Si $Y \sim N(\Gamma, V \otimes \Sigma), A_{n \times r}, B_{n \times q}$ matrices, entonces

$$
\operatorname{cov}(Y B, Y A)=B^{\prime} V A \otimes \Sigma, \quad \operatorname{cov}(Y A, Y B)=A^{\prime} V B \otimes \Sigma .
$$

- $Y^{\prime}=\left(Y^{1}, \ldots, Y^{p}\right), \quad \operatorname{var}\left(Y^{\prime}\right)=\Sigma \otimes V \quad \operatorname{cov}\left(Y^{\prime}, Y\right)=\Sigma \dot{\otimes} V$

$$
\operatorname{var}(Y)=V \otimes \Sigma \quad \operatorname{cov}\left(Y, Y^{\prime}\right)=V \dot{\otimes} \Sigma .
$$


- $\operatorname{cov}\left(A Y^{\prime}, Y B\right)=\Sigma \dot{\otimes} A V B \quad \operatorname{cov}\left(Y B, A Y^{\prime}\right)=(A V B)^{\prime} \dot{\otimes} \Sigma$ $\operatorname{cov}(Y C, Y D)=C^{\prime} V D \otimes \Sigma \quad \operatorname{cov}\left(E Y^{\prime}, F Y^{\prime}\right)=\Sigma \otimes E V F^{\prime}$.

- $\operatorname{cov}(K Y A, H Y B)=A^{\prime} V B \otimes K \Sigma H^{\prime}$.

- Calculemos la covarianza entre dos formas cuadráticas mixtas.

$$
\begin{aligned}
{[t] l A } & =Y A Y^{\prime}-\Gamma A \Gamma^{\prime}-\Sigma \operatorname{tr}(A V) \\
& =\sum_{\alpha=1}^{n} \sum_{\beta=1}^{n}\left[a_{\alpha \beta} X_{\alpha} X_{\beta}^{\prime}+\delta_{\alpha \beta} \nu_{\alpha} X_{\beta}^{\prime}+\delta_{\alpha \beta} X_{\alpha} \nu_{\beta}^{\prime}-\delta_{\alpha \beta}(A V)_{\alpha \beta} \Sigma\right] \\
K & =Y^{\prime} K Y-\Gamma^{\prime} K \Gamma-V \operatorname{tr}(K \Sigma) \\
& =\sum_{s=1}^{p} \sum_{t=1}^{p}\left[k_{s t} X^{s} X^{t \prime}+\delta_{s t} \lambda_{s} X^{t \prime}+\delta_{s t} X^{s} \lambda_{t}^{\prime}-\delta_{s t}(\Sigma K)_{s t} V\right]
\end{aligned}
$$

donde $\nu=\left(\nu_{1}, \ldots, \nu_{n}\right)=\Gamma A, X_{\alpha}=Y_{\alpha}-\mu_{\alpha}, \lambda=\left(\lambda_{1}, \ldots, \lambda_{p}\right)=K \Gamma^{\prime}, X^{s}=Y^{s}-\pi_{s}$, $\Gamma=\left(\mu_{1}, \ldots, \mu_{n}\right), \Gamma^{\prime}=\left(\pi_{1}, \ldots, \pi_{p}\right)$.

$$
\begin{aligned}
E(A \ddot{\otimes} K)= & \sum_{\alpha \beta s t} E\left[a_{\alpha \beta} k_{s t} X_{\alpha} X_{\beta} \ddot{\otimes} X^{s} X^{t \prime}+\delta_{\alpha \beta} k_{s t} \nu_{\alpha} X_{\beta}^{\prime} \ddot{\otimes} X^{s} X^{t \prime}+\delta_{\alpha \beta} k_{s t} X_{\alpha} \nu_{\beta}^{\prime} \ddot{\otimes} X^{s} X^{t \prime}\right. \\
& -\delta_{\alpha \beta}(A V)_{\alpha \beta} k_{s t} \Sigma \ddot{\otimes} X^{s} X^{t \prime}+a_{\alpha \beta} \delta_{s t} X_{\alpha} X_{\beta}^{\prime} \ddot{\otimes} \lambda_{s} X^{t \prime}+\delta_{\alpha \beta} \delta_{s t} \nu_{\alpha} X_{\beta}^{\prime} \ddot{\otimes} \lambda_{s} X^{t \prime} \\
& +\delta_{\alpha \beta} \delta_{s t} X_{\alpha} \nu_{\beta}^{\prime} \ddot{\otimes} \lambda_{s} X^{t \prime}+a_{\alpha \beta} \delta_{s t} X_{\alpha} X_{\beta}^{\prime} \ddot{\otimes} X^{s} \lambda_{t}^{\prime}+\delta_{\alpha \beta} \delta_{s t} \nu_{\alpha} X_{\beta}^{\prime} \ddot{\otimes} X^{s} \lambda_{t}^{\prime} \\
& \left.+\delta_{\alpha \beta} \delta_{s t} X_{\alpha} \nu_{\beta}^{\prime} \ddot{\otimes} X^{s} \lambda_{t}^{\prime}-a_{\alpha \beta} \delta_{s t}(\Sigma K)_{s t} X_{\alpha} X_{\beta}^{\prime} \ddot{\otimes} V+\delta_{\alpha \beta} \delta_{s t}(A V)_{\alpha \beta}(\Sigma K)_{s t} \Sigma \ddot{\otimes} V\right]
\end{aligned}
$$

pues la esperanza de los términos de orden uno es nula.

- $\sum_{\alpha \beta s t} a_{\alpha \beta} k_{s t} E\left(X_{\alpha} X_{\beta}^{\prime} \ddot{\otimes} X^{s} X^{t \prime}\right)=\sum_{\alpha \beta s t} a_{\alpha \beta} k_{s t} E\left(X_{\alpha} X^{s \prime} \otimes X_{\beta} X^{t \prime}\right)=2 \Sigma K \Sigma \ddot{\otimes} V A V+$ $\operatorname{tr}(A V) \operatorname{tr}(\Sigma K) \Sigma \ddot{\otimes} V+M^{*} \ddot{\otimes} D_{A}-2 \Sigma K \Sigma \ddot{\otimes} V D_{A} V-\operatorname{tr}(K \Sigma) \Sigma \ddot{\otimes} D_{A V} V$, donde $D_{A}$ es la matriz diagonal que tiene los elementos $a_{i i}$ en la diagonal, la entrada $\bar{M}_{i s k t}=$ $E\left(x_{i \alpha} x_{s \alpha} x_{k \alpha} x_{t \alpha}\right)$ de $\bar{M} p^{2} \times p^{2}$, de modo que $M_{i k}^{*}=\sum_{s t} k_{s t} \bar{M}_{k t}^{i s}=\operatorname{tr}\left(K \bar{M}^{i k}\right)$, $\bar{M}=\left(\begin{array}{ccc}\bar{M}^{11} & \cdots & \bar{M}^{1 p} \\ \vdots & \ddots & \vdots \\ \bar{M}^{p 1} & \cdots & \bar{M}^{p p}\end{array}\right)$.

En efecto, consideremos la entrada $i j k l$ de $E\left(X_{\alpha} X^{s \prime} \otimes X_{\beta} X^{t \prime}\right)$ i.e.

$$
\begin{array}{rl}
\sum_{\alpha \beta s t} a_{\alpha \beta} k_{s t} & E\left(X_{i \alpha} X_{s j} X_{k \beta} X_{t l}\right)=\sum_{\alpha \beta s t} a_{\alpha \beta} k_{s t} v_{\alpha j} \sigma_{i s} v_{\beta l} \sigma_{k t}+\sum_{\alpha \beta s t} a_{\alpha \beta} k_{s t} v_{\alpha \beta} \sigma_{i k} v_{j l} \sigma_{s t} \\
& +\sum_{\alpha \beta s t} a_{\alpha \beta} k_{s t} v_{\alpha l} \sigma_{i t} v_{j \beta} \sigma_{s k}+\sum_{s t} a_{j j} \delta_{j l} k_{s t} \bar{M}_{i s k l}-\sum_{\alpha s t} a_{\alpha \alpha} k_{s t} v_{\alpha j} \sigma_{i s} v_{\alpha l} \sigma_{k t} \\
& -\sum_{\alpha s t} a_{\alpha j} k_{s t} v_{\alpha j} \sigma_{i k} v_{j l} \sigma_{s t}-\sum_{\alpha s t} a_{\alpha \alpha} k_{s t} v_{\alpha l} \sigma_{i t} v_{j \alpha} \sigma_{s k} \\
= & (\Sigma K \Sigma)_{i k}(V A V)_{j l}+\operatorname{tr}(A V) \operatorname{tr}(\Sigma K) \sigma_{i k} v_{j l}+(\Sigma K \Sigma)_{i k}(V A V)_{j l}+a_{j j} \delta_{j l} \sum_{s t} k_{s t} \bar{M}_{i s k t} \\
& -\left(V D_{A} V\right)_{j l}(\Sigma K \Sigma)_{i k}-(A V)_{j j} v_{j l} \operatorname{tr}(K \Sigma) \sigma_{i k}-(V A V)_{j l}(\Sigma K \Sigma)_{i k}
\end{array}
$$


- $\sum_{\alpha \beta s t} \delta_{\alpha \beta} k_{s t} E\left(\nu_{\alpha} X_{\beta}^{\prime} \ddot{\otimes} X^{s} X^{t \prime}\right)=\sum_{\alpha \beta s t} \delta_{\alpha \beta} k_{s t} E\left(\nu_{\alpha} X^{s \prime} \otimes X_{\beta} X^{t \prime}\right)=\sum_{\alpha s t} k_{s t} \nu_{\alpha} \otimes$ $\bar{M}_{s t}^{\cdot \alpha}$

- $\sum_{\alpha \beta s t} \delta_{\alpha \beta} k_{s t} E\left(X_{\alpha} X^{s \prime} \otimes \nu_{\beta} X^{t \prime}\right)=\sum_{\alpha s t} k_{s t} \bar{M}_{s t}^{\cdot \alpha} \otimes \nu_{\alpha}$

- $\sum_{\alpha \beta s t} \delta_{\alpha \beta}(A V)_{\alpha \beta} k_{s t} \Sigma \ddot{\otimes} E\left(X^{s} X^{t \prime}\right)=\operatorname{tr}(A V) \operatorname{tr}(\Sigma K) \Sigma \ddot{\otimes} V$

- $\sum_{\alpha \beta s t} a_{\alpha \beta} \delta_{s t} E\left(X_{\alpha} X_{\beta}^{\prime} \ddot{\otimes} \lambda_{s} X^{t \prime}\right)=\sum_{\alpha \beta t} a_{\alpha \beta} \lambda_{t}^{\prime} \otimes\left(\bar{M}_{\alpha \beta}^{\cdot s}\right)^{\prime}$

- $\sum_{\alpha \beta s t} \delta_{\alpha \beta} \delta_{s t} E\left(\nu_{\alpha} X_{\beta}^{\prime} \ddot{\otimes} \lambda^{s} X^{t \prime}\right)=\sum_{\alpha \beta s t} \delta_{\alpha \beta} \delta_{s t} E\left(\nu_{\alpha} \lambda_{s}^{\prime} \otimes X_{\beta} X^{t \prime}\right)=\Gamma A V \otimes \Sigma K \Gamma^{\prime}$

$-\sum_{\alpha \beta s t} \delta_{\alpha \beta} \delta_{s t} E\left(X_{\alpha} \nu_{\beta}^{\prime} \ddot{\otimes} \lambda^{s} X^{t \prime}\right)=\Sigma K \Gamma^{\prime} \dot{\otimes} \Gamma A V$

- $\sum_{\alpha \beta s t} a_{\alpha \beta} \delta_{s t} E\left(X_{\alpha} X_{\beta}^{\prime} \ddot{\otimes} X^{s} \lambda_{t}^{\prime}\right)=\sum_{\alpha \beta t} a_{\alpha \beta}\left(\bar{M}_{\alpha \beta}^{\cdot t}\right)^{\prime} \otimes \lambda_{t}^{\prime}$

- $\sum_{\alpha \beta s t} \delta_{\alpha \beta} \delta_{s t} E\left(\nu_{\alpha} X_{\beta}^{\prime} \ddot{\otimes} X^{s} \lambda_{t}^{\prime}\right)=\Gamma A V \dot{\otimes} \Sigma K \Gamma^{\prime}$

$-\sum_{\alpha \beta s t} \delta_{\alpha \beta} \delta_{s t} E\left(X_{\alpha} \nu_{\beta}^{\prime} \ddot{\otimes} X^{s} \lambda_{t}^{\prime}\right)=\Sigma K \Gamma^{\prime} \otimes \Gamma A V$

$-\sum_{\alpha \beta s t} a_{\alpha \beta} \delta_{s t}(K \Sigma)_{s t} E\left(X_{\alpha} X_{\beta}^{\prime}\right) \ddot{\otimes} V=\operatorname{tr}(A V) \operatorname{tr}(\Sigma K) \Sigma \ddot{\otimes} V$

$-\sum_{\alpha \beta s t} \delta_{\alpha \beta}(A V)_{\alpha \beta} \delta_{s t}(K \Sigma)_{s t} \Sigma \ddot{\otimes} V=\operatorname{tr}(A V) \operatorname{tr}(\Sigma K) \Sigma \ddot{\otimes} V$.

Así tenemos

$$
\begin{aligned}
& \operatorname{cov}\left(Y A Y^{\prime}, Y^{\prime} K Y\right)=2 \Sigma K \Sigma \ddot{\otimes} V A V+\bar{M}^{*} \ddot{\otimes} D_{A}-2 \Sigma K \Sigma \ddot{\otimes} V D_{A} V-\operatorname{tr}(K \Sigma) \Sigma \ddot{\otimes} D_{A} V \\
& \quad+\Gamma A V \dot{\otimes} \Sigma K \Gamma^{\prime}+\Sigma K \Gamma^{\prime} \dot{\otimes} \Gamma A V+\Gamma A V \otimes \Sigma K \Gamma^{\prime}+\Sigma K \Gamma^{\prime} \otimes \Gamma A V+\sum_{\alpha s t} k_{s t} \nu_{\alpha} \otimes \bar{M}_{s t}^{\cdot \alpha} \\
& \quad+\sum_{\alpha s t} k_{s t} \bar{M}_{s t}^{\cdot \alpha} \otimes \nu_{\alpha}+\sum_{\alpha \beta s} a_{\alpha \beta}\left(\bar{M}_{\alpha \beta}^{\cdot s}\right)^{\prime} \otimes \lambda_{s}^{\prime}+\sum_{\alpha \beta s} a_{\alpha \beta} \lambda_{s}^{\prime} \otimes\left(\bar{M}_{\alpha \beta}^{\cdot s}\right)^{\prime} .
\end{aligned}
$$

\begin{tabular}{|l|l|}
\hline$E\left(Y A Y^{\prime}\right)=\Gamma A \Gamma^{\prime}+\Sigma \operatorname{tr}(A V)$ & $E\left(Y^{\prime} H Y\right)=\Gamma^{\prime} H \Gamma+V \operatorname{tr}(\Sigma H)$ \\
\hline $\operatorname{cov}\left(Y A Y^{\prime}, Y B\right)=\Gamma A V B \otimes \Sigma+\Sigma \dot{\otimes} \Gamma A V B+\bar{M}_{3} \otimes a^{\prime} B$ & $\operatorname{cov}\left(Y^{\prime} H Y, Y^{\prime} K\right)=\Gamma^{\prime} H \Sigma K \otimes V+V \dot{\otimes} \Gamma^{\prime} H \Sigma K+\bar{N}_{3} \otimes h^{\prime} K$ \\
\hline $\operatorname{cov}\left(Y A Y^{\prime}, Y B Y^{\prime}\right)=\operatorname{tr}(A V B V)(\Sigma \otimes \Sigma+\Sigma \dot{\otimes} \Sigma)+$ & $\operatorname{cov}\left(Y^{\prime} H Y, Y^{\prime} K Y\right)=\operatorname{tr}(H \Sigma K \Sigma)(V \otimes V+V \dot{\otimes})+$ \\
$\Sigma \otimes \Gamma A V B \Gamma^{\prime}+\Sigma \dot{\otimes} \Gamma A V B \Gamma^{\prime}+\Gamma A V B \Gamma^{\prime} \otimes \Sigma+$ & $V \otimes \Gamma^{\prime} H \Sigma K \Gamma+V \dot{\otimes} \Gamma^{\prime} H \Sigma K \Gamma+\Gamma^{\prime} H \Sigma K \Gamma \otimes V+$ \\
$\Gamma A V B \Gamma^{\prime} \dot{\otimes} \Sigma+a^{\prime} b\left(\bar{M}_{4}-\Sigma \otimes \Sigma-\Sigma \dot{\otimes} \Sigma-\Sigma \ddot{\otimes} \Sigma\right)+$ & $\Gamma^{\prime} H \Sigma K \Gamma \dot{\otimes} V+h^{\prime} k\left(\bar{N}_{4}-V \otimes V-V \dot{\otimes} V-V \ddot{\otimes} V\right)+$ \\
$\bar{M}_{3}^{\prime} \otimes a^{\prime} B \Gamma^{\prime}+a^{\prime} B \Gamma^{\prime} \otimes \bar{M}_{3}^{\prime}+\bar{M}_{3} \otimes \Gamma A b+\Gamma A b \otimes \bar{M}_{3}$ & $\bar{N}_{3}^{\prime} \otimes h^{\prime} K \Gamma+h^{\prime} K \Gamma \otimes \bar{N}_{3}^{\prime}+\bar{N}_{3} \otimes \Gamma^{\prime} H k+\Gamma H k \otimes \bar{N}_{3}$ \\
\hline $\operatorname{cov}\left(A Y^{\prime} K, H Y B\right)=K^{\prime} \Sigma H^{\prime} \dot{\otimes} A V B$ & $\operatorname{cov}\left(H Y B, A Y^{\prime} K\right)=B^{\prime} V A^{\prime} \dot{\otimes} H \Sigma K$ \\
\hline $\operatorname{cov}(K Y A, H Y B)=A^{\prime} V B \otimes K \Sigma H^{\prime}$ & $\operatorname{cov}\left(A Y^{\prime} K, B Y^{\prime} H\right)=K \Sigma H^{\prime} \otimes A^{\prime} V B$ \\
\hline
\end{tabular}




\section{Referencias}

[1] Anderson, T.W. (1958) An Introduction to Multivariate Statistical Analysis. John Wiley \& Sons, New York.

[2] Barra, J.R. (1971) Notions Fondamentales de Statistique Mathématique. Dunod, Paris.

[3] Muirhead, R.J. (1982) Aspects of Multivariate Statistical Theory. John Wiley \& Sons, New York.

[4] Poltronieri, J. (1987) "Algunas consideraciones sobre las formas cuadráticas y las formas lineales", Uniciencia, 4(1,2): 69-75.

[5] Poltronieri, J. (1987) "Algunas propiedades útiles en estadística", Uniciencia, 4(1,2): $59-67$.

[6] Poltronieri, J. (1988) "Estudio de formas cuadráticas y formas lineales en el caso multivariado", Memorias IV Simposio de Métodos Matemáticos Aplicados a las Ciencias, B. Montero \& J. Poltronieri (eds.), Editorial de la Universidad de Costa Rica: 165173.

[7] Poltronieri, J. (1988) "Sobre la covarianza de formas cuadráticas y formas lineales", Memorias IV Simposio de Métodos Matemáticos Aplicados a las Ciencias, B. Montero \& J. Poltronieri (eds.), Editorial de la Universidad de Costa Rica: 174-180.

[8] Poltronieri, J. (1995) "Contribución al estudio de formas cuadráticas en estadística multivariada", Memorias Simposio de Métodos Matemáticos Aplicados a las Ciencias, Inst. Tecnol. de C.R., J. Trejos (ed.): 133-142.

[9] Searle, S.R. (1971) Linear Models. John Wiley \& Sons, New York.

[10] Styan, G.P.H. (1969) Notes on the distribution of quadratic formes in singular normal variables. Technical Report 122, University of Minnesota, Minneapolis, Minn. 